30-40\% (50\% if both parents are affected). (See Bird TD. Epilepsia 1987;28 (Suppl 1):S71-81, reviewed in Progress in Pediatric Neurology I, PNB Publ, 1991;pp15-16). In my own series of FS patients, a family history of FS is found in $17 \%$ of cases. The incidence is similar in those complicated by nonfebrile seizures, and an inherited tendency to nonfebrile seizures is equally prevalent. Recurrent nonfebrile seizures, especially generalized tonic-clonic epilepsy, among children with FS are reported in 20\% (average) of 4500 patients in 35 publications between 1929 and 1964 . The average familial incidence of epilepsy in the total was $15 \%$. Analyses of these case reports suggest that the genetically determined factors that may result in a low threshold to FS are associated with an equally prevalent genetic predisposition to nonfebrile seizures (Millichap JG. Febrile Convulsions. New York, Macmillan, 1968). These previous reports are in agreement with the concept of the GEFS+ syndrome, and families identified warrant genetic analysis.

\title{
TEMPERATURE, AGE, AND FEBRILE SEIZURE RECURRENCE
}

Factors that predict recurrence of a seizure during a subsequent fever episode were studied in 230 children included in a randomized placebo controlled trial of ibuprofen prophylaxis treatment at the Sophia and Juliana Children's Hospitals, Rotterdam, the Netherlands. There were 67 febrile seizure (FS) recurrences in a total of 509 fever episodes; 35 (52\%) occurred within 2 hours of fever onset and $32(48 \%)$ after more than 2 hours of fever. The median temperature with recurrences early in the fever episode was lower $\left(39.3^{\circ} \mathrm{C}\right)$ than that registered during delayed FS recurrences $\left(40.0^{\circ} \mathrm{C}\right)$. The risk of FS recurrence increases with temperature at fever of onset and during the febrile episode and decreases with age. A child aged 1 year with a $30 \%$ recurrence risk has a 5 to $10 \%$ risk at 4 years. (van Stuijvenberg M, Steyerberg EW, Derksen-Lubsen G, Moll HA. Temperature, age, and recurrence of febrile seizure. Arch Pediatr Adolesc Med Dec 1998;152:1170-1175). (Respond: Henriette A Moll MD, PhD, Sophia Children's Hospital, Rm Sp 1543, Dr Molewaterplein 60, 3015 GJ Rotterdam, the Netherlands).

COMMENT. Recurrent febrile seizures occur within 2 hours of onset of a fever episode in $50 \%$ of cases. Seizures recurring after 2 hours of fever are associated with higher temperature levels than those within the first 2 hours of fever. The risk of FS recurrence with subsequent fever decreases with age and increases with temperature at fever onset and during fever episodes.

A threshold convulsive temperature, suggested by this report, was first demonstrated in laboratory and clinical studies in Jan 1959, exactly forty years ago (Millichap JG. Studies in febrile seizures. I. Height of body temperature as a measure of the febrile seizure threshold. Pediatrics 1959;23:76-85. Idem. Febrile Convulsions. New York, Macmillan, 1968). The rapidity of rise of body temperature, previously proposed as an essential mechanism for febrile seizures, was non-contributory. Factors found to modify the threshold convulsive temperature include age, brain maturation, electrolyte imbalance, and various medications. A postictal elevation in seizure threshold requires a relatively higher body temperature to induce a second seizure.

\section{GENETICS OF BENIGN ROL.ANDIC EPILEPSY}

Twenty-two nuclear families with benign epilepsy of childhood with centrotemporal spikes (BECTS, rolandic epilepsy) were analyzed by DNA linkage studies at the Department of Neuropediatrics, University of Kiel, Germany. Screening of all chromosomal regions known to harbor neuronal nicotinic acetylcholine receptor (AChR) subunit genes found evidence for linkage of BECTS 